\section{Acuidade no diagnóstico da laringomalácia através da nasofibrolaringoscopia}

\author{
Tania Mara Assis Lima ', Denise Utsch Gonçalves \\ ${ }^{2}$, Lucas V. Gonçalves ${ }^{3}$, Paulo Augusto C. Reis ${ }^{4}$, \\ Angela Beatriz S. Lana ${ }^{5}$, Fernando F. Guimarães ${ }^{6}$
}

\title{
Flexible nasolaryngoscopy accuracy in laryngomalacia diagnosis
}

\author{
Palavras-chave: diagnóstico, laringe, laringomalácia, \\ laringoscopia.
}

Keywords: diagnosis, larynx, abnormalities, laryngoscopy.

\section{Resumo / Summary}

\begin{abstract}
A laringomalácia é a causa mais comum de estridor na infância. O diagnóstico é estabelecido através do quadro clínico associado aos achados laringoscópicos analisados de forma dinâmica. Objetivo: Avaliar a confiabilidade no diagnóstico da laringomalácia através da nasofibrolaringoscopia. Forma de Estudo: Concordância para avaliar a confiabilidade de um teste diagnóstico (clínico). Material e Método: Dezoito pacientes com diagnóstico prévio de laringomalácia a partir dos dados clínicos, epidemiológicos e anatômicos foram avaliados. O exame videolaringoscópico desses pacientes foi comparado com relação ao índice de concordância de quatro diferentes examinadores, através da análise cega de um em relação ao exame do outro observador. No protocolo de avaliação, os critérios considerados para o diagnóstico foram: basculamento anterior das aritenóides; epiglote em ômega; pregas ariepiglóticas curtas; basculamento posterior da epiglote; visão ou não da prega vocal; edema da laringe posterior. Os observadores variavam em relação à experiência profissional. Comparou-se a média de concordância de todos os parâmetros considerados. O padrão ouro foi o consenso no exame dos quatro observadores. Resultados: A média de concordância foi de 88,2\%. Discussão e Conclusão: A nasofibrolaringoscopia é um método diagnóstico de boa acuidade, uma vez que a experiência do profissional não interferiu no resultado do exame.
\end{abstract}

\begin{abstract}
L aryngomalacia is the most common cause of stridor in infancy. Diagnosis is established by associating the clinical manifestations with laryngoscopic findings in a dynamic form. Aim: To analyze diagnostic accuracy of laryngomalacia through flexible nasolaryngoscopy performed by four different examiners. Form of studying: Comparison of diagnostic tests (clinical study). Material and method: A protocol of videolaryngoscopic evaluation for patients with laryngomalacia was created encompassing the following items: anterior displacement of the arytenoids; omega-shaped epiglottis; short aryepiglottic folds; posterior displacement of epiglottis; vocal folds being visible or not; edema of the posterior larynx. Four different examiners analyzed the videolaryngoscopic examinations of 18 patients with established diagnosis of laryngomalacia previously established by clinical data, epidemiology and anatomical traits. The four observers knew previously that the patients had laryngomalacia and which criteria should be analyzed in the evaluation protocol. Observers were unaware of the results each one found. Results: diagnostic agreement average considering all parameters evaluated was of $88.2 \%$. Discussion/Conclusion: Dynamic flexible nasolaryngoscopy is a proven diagnostic method, regardless of physician experience.
\end{abstract}

${ }^{1}$ Doutora em Otorrinolaringologia, Prof. Adjunta do Dept. Otorrinolaringologia FM UFMG

${ }^{2}$ Doutora, Profa. adjunta do Departamento de Oftalmologia e Otorrinolaringologia da Fac. Medicina da UFMG.

${ }^{3}$ Médico residente do Serviço de Otorrinolaringologia do Hospital das Clínicas da UFMG, Residente de Otorrinolaringologia do Hospital das Clínicas da UFMG.

${ }^{4}$ Médico, Residente de Otorrinolaringologia do Hospital das Clínicas da UFMG.

5 Médica, Residente em Otorrinolaringologia do Hospital das Clínicas da UFMG.

${ }^{6}$ Médico, Residente em Otorrinolaringologia do Hospital das Clínicas da UFMG. Faculdade de Medicina da UFMG e Hospital das Clínicas da UFMG

Endereço para correspondência: Tânia Mara Assis Lima - Rua do Ouro 229/502 Bairro Serra Belo Horizonte MG 30220-000.

Este artigo foi submetido no SGP (Sistema de Gestão de Publicações) da RBORL em 8 de novembro de 2006. cod. 3501

Artigo aceito em 2 de janeiro de 2008. 


\section{INTRODUÇÃO}

Laringomalácia é a causa mais comum de estridor na infância, sendo responsável por 65 a 75\% dos casos de estridor ${ }^{1}$. Acomete mais o sexo masculino numa proporção de 2:1. Essa condição é determinada pela incapacidade laríngea de manter seu lúmen aberto durante a inspiração. O estridor inspiratório é a principal manifestação da doença ${ }^{2}$ que é, na maioria das vezes, auto-limitada e se resolve nos primeiros 12 a 18 meses de vida. Contudo, em 10\% dos casos é necessária a abordagem cirúrgica, uma vez que a obstrução respiratória pode levar a quadros de dispnéia grave, disfagia, retardo no crescimento e apnéia obstrutiva do sono ${ }^{1}$.

Sua etiologia ainda não é conhecida. Várias teorias tentam explicar a etiopatogenia da laringomalácia. A mais aceita, atualmente, diz ser a laringomalácia resultante de uma imaturidade neuromuscular levando a uma hipotonia supraglótica e acarretando a flacidez dessas estruturas durante a inspiração ${ }^{2}$. Outra teoria aponta para uma diminuição da sustentação do arcabouço cartilaginoso. A hipotonia da musculatura laríngea também é citada como possível mecanismo etiopatogênico.

O diagnóstico é baseado na história clínica e epidemiológica de recém-nascidos e lactentes com estridor inspiratório, que piora com agitação ou choro e melhora durante o sono. Tradicionalmente, a laringoscopia direta é o exame padrão ouro para o diagnóstico do estridor ${ }^{3-5}$. Como a laringomalácia é o diagnóstico mais comum de estridor e, geralmente, a causa mais benigna, a avaliação dinâmica da laringe através da nasolaringoscopia com endoscópio flexível vem sendo empregada com bons resultados, apesar de não substituir a laringoscopia direta com endoscópio rígido nos casos indicados. ${ }^{6} \mathrm{~A}$ avaliação da imagem endoscópica tem sido, contudo, pouco sistematizada até o presente momento, com fatores de confundimento relacionados à experiência do observador e à variação anatômica inerente do ser humano. Por isso, resta estabelecer de forma controlada quais alterações anatômicas demonstraram ser mais constantes quando avaliadas pela nasofibrolaringoscopia, estabelecendo parâmetros que devem ser avaliados em todo caso com suspeita de laringomalácia. Isto não parece ter sido realizado até o presente momento.

O objetivo desse estudo foi estabelecer a acuidade diagnóstica da videolaringoscopia para o diagnóstico da laringomalácia, quando realizado por observadores com experiência clínica variável em laringologia.

\section{MATERIAL E MÉTODO}

Trata-se de um estudo de concordância para avaliar a confiabilidade de um teste diagnóstico. O projeto foi avaliado pelo Comitê de Ética em Pesquisa da UFMG sob número 571/07.

Dezoito pacientes com diagnóstico previamente estabelecido de laringomalácia a partir dos dados clínicos, epidemiológicos e anatômicos foram avaliados. O exame videolaringoscópico desses pacientes foi comparado com relação ao índice de concordância de quatro observadores diferentes. O protocolo de avaliação videolaringoscópica dos pacientes foi baseado nas alterações anatômicas mais freqüentemente observadas nessa enfermidade ${ }^{2}$. Os parâmetros considerados foram:

1) basculamento anterior das aritenóides durante a inspiração;

2) epiglote tubular (em ômega) que se colaba na inspiração;

3) pregas ariepiglóticas curtas;

4) basculamento posterior da epiglote;

5) visão ou não das pregas vocais;

6) presença de edema das estruturas laríngeas posteriores. Os exames foram realizados com nasolaringoscópio flexível e gravados em vídeo.

Os observadores apresentavam experiência clínica em laringologia infantil que variava de 2 anos (observadores 1, 2 e 3) a 15 anos (observador 4). Todos sabiam previamente que estavam analisando os exames de pacientes com diagnóstico de certeza de laringomalácia e também sabiam quais os critérios anatômicos deveriam ser considerados.

As análises foram realizadas de forma cega de um em relação ao exame de outro observador. Posteriormente, todos os observadores analisaram conjuntamente os exames laringoscópicos chegando a um consenso, considerado como referência. Logo, para estabelecer-se o padrão ouro considerou-se o consenso dos exames e não o número total de doentes. Interessou verificar a concordância entre as alterações estabelecidas pelos observadores em relação ao consenso (padrão ouro). Na comparação, cada observador foi avaliado quanto ao índice de acertos em relação ao padrão ouro.

\section{RESULTADOS}

A análise das dezoito laringoscopias realizadas por cada observador e o consenso das avaliações estão registradas na Tabela 1. A concordância média de todos os parâmetros considerados em relação à análise consensual dos quatro observadores foi de $88,2 \%$. Os dados dessa análise estão especificados na Tabela 2.

Quanto à análise individual, o observador 4, com 15 anos de experiência clínica, apresentou maior discordância em relação ao parâmetro "edema de laringe posterior" em relação aos observadores com 2 anos de experiência clínica (Tabela 2). 
Tabela 1. Parâmetros considerados no exame videolaringoscópico realizado por quatro observadores e o consenso dessa análise no diagnóstico de 18 pacientes com laringomalácia.

\begin{tabular}{|c|c|c|c|c|c|}
\hline \multirow{2}{*}{ Parâmetros no exame videolaringoscópico } & \multicolumn{4}{|c|}{ Observadores } & \multirow{2}{*}{ Consenso } \\
\hline & 1 & 2 & 3 & 4 & \\
\hline Basculamento anterior das aritenóides & 12 & 14 & 15 & 16 & 16 \\
\hline Epiglote em ômega & 11 & 14 & 13 & 11 & 11 \\
\hline Pregas ari-epiglóticas curtas & 17 & 18 & 17 & 17 & 18 \\
\hline Basculamento posterior da epiglote & 1 & 2 & 1 & 1 & 1 \\
\hline Não ver pregas vocais & 5 & 3 & 5 & 4 & 4 \\
\hline Edema da laringe posterior & 14 & 16 & 13 & 13 & 15 \\
\hline
\end{tabular}

Tabela 2. Índice de concordância de quatro observadores em relação ao resultado consensual de 16 exames videolaringoscópicos de pacientes com laringomalácia.

\begin{tabular}{|c|c|c|c|c|c|}
\hline \multirow{2}{*}{ Parâmetros no exame videolaringoscópico } & \multicolumn{4}{|c|}{ Avaliação dos observadores } & \multirow{2}{*}{$\begin{array}{c}\text { Índice de } \\
\text { concordância }\end{array}$} \\
\hline & 1 & 2 & 3 & 4 & \\
\hline Basculamento anterior das aritenóides & $12 / 16$ & $14 / 16$ & $15 / 16$ & $16 / 16$ & $88,8 \%$ \\
\hline \multirow{2}{*}{ Epiglote em ômega } & $11 / 11$ & $7 / 11$ & $9 / 11$ & $11 / 11$ & \multirow{2}{*}{$86,0 \%$} \\
\hline & $(100 \%)$ & $(63 \%)$ & $(81 \%)$ & $(100 \%)$ & \\
\hline Pregas ari-epiglóticas curtas & $(94,4 \%)$ & $(100 \%)$ & $(94,4 \%)$ & $(94,4 \%)$ & $95,8 \%$ \\
\hline \multirow{2}{*}{ Basculamento posterior da epiglote } & $1 / 1$ & $0,5 / 1$ & $1 / 1$ & $1 / 1$ & \multirow[t]{2}{*}{$87,5 \%$} \\
\hline & $(100 \%)$ & $(50 \%)$ & $(100 \%)$ & $(100 \%)$ & \\
\hline \multirow{2}{*}{ Não ver pregas vocais } & $3 / 4$ & $3 / 4$ & $3 / 4$ & $4 / 4$ & \multirow[t]{2}{*}{$81,4 \%$} \\
\hline & $(75 \%)$ & $(75 \%)$ & $(75 \%)$ & $(100 \%)$ & \\
\hline
\end{tabular}

\section{DISCUSSÃO}

A nasolaringoscopia é um exame muito importante na prática otorrinolaringológica, sendo cada vez mais indicado na avaliação de afecções das vias aéreas superiores. Por ser um exame que depende da análise do observador, está sujeito a variações de percepção relacionadas, sobretudo, à experiência clínica do examinador.

Em relação ao diagnóstico de laringomalácia, a média de concordância dos parâmetros avaliados foi de $88,2 \%$ entre os observadores. O parâmetro mais confiável foi "pregas ariepiglóticas curtas", cuja concordância foi de 95,8\%. O menos confiável foi "não visualização das pregas vocais”, em que a concordância foi de 81,4\%. Não conseguir ver as pregas vocais na avaliação videolaringoscópica tem sido relacionado ao prognóstico, pois alguns autores utilizam esse dado, além do quadro clínico, para indicar o tratamento cirúrgico ${ }^{7}$.

Neste estudo, o índice de concordância considerando todos os parâmetros avaliados, mostrou-se elevado, demonstrando que o exame é confiável para o diagnóstico de laringomalácia, mesmo quando realizado por profissionais com experiência escassa em laringologia.

Dois parâmetros merecem ser ressaltados:

1) visão ou não das pregas vocais, pois os observadores com menor experiência em laringologia tiveram dificuldade em identificá-lo e este é um dos parâmetros considerados na indicação cirúrgica ${ }^{7}$;

2) presença de edema das estruturas laríngeas posteriores $^{8}$, pois foi identificado com maior facilidade pelo observador com maior experiência clínica.

\section{CONCLUSÃO}

A nasofibrolaringoscopia é um bom exame para o diagnóstico de laringomalácia, com sensibilidade de 88,2\% independentemente da experiência clínica do observador. O parâmetro mais confiável foi avaliação de pregas ariepiglóticas curtas e o menos confiável foi ver ou não as pregas vocais. Para casos com possibilidade de indicação cirúrgica e para avaliar a presença de edema nas estruturas laríngeas posteriores a experiência clínica em laringologia infantil é importante. 


\section{REFERÊNCIAS BIBLIOGRÁFICAS}

1.Olney DR, Greinwald JH, Smith RJ, Bauman N. Laryngomalacia and its Treatment. Laryngoscope 1999;109(11):1770-5.

2.Duprat AD, Campos CAH, Costa HOO. Tratado de Otorrinolaringologia (vol 4) - 1a ed. São Paulo: Roca; 2003; 440-50.

3.Holinger LD. Diagnostic endoscopy of the pediatric airway. Laryngoscope 1989;99:346-48.

4.Berkowitz RG. Neonatal upper airway assessment by awake flexible laryngoscopy. Ann Otol Rhinol Laryngol 1998;107(1):75-80.

5.Moumoulidis I, Gray RF, Wilson T. Outpatient fiber-optic laryngoscopy for stridor in children and infants. Eur Arch Otorhinolaryngol 2005:262(3):204-7.
6.Waters KA, Woo P, Mortelliti AJ. Assessment of the infant airway with videorecorded flexible laryngoscopy and the objective analyses of vocal fold abduction. Otolaryngol Head Neck Surg 1996;114(4):55461

7.Zalzal GH, Anon JB, Cotton RT. Epigottoplasty for the treatment of laryngomalacia. Ann Otol Rhinol Laryngol 1987;96:72-6.

8.Ungkanot K, Friedman E, Sulek M. Retrospective analyses of airway endoscopy in patients less than 1-month old. Laryngoscope 1998;108(11):1724-28. 